\title{
Assessing the Survival Benefit of Surgery and Various Types of Radiation Therapy for Treatment of Hepatocellular Carcinoma: Evidence from the Surveillance, Epidemiology, and End Results Registries
}

This article was published in the following Dove Press journal:

Journal of Hepatocellular Carcinoma

Fuyan Shi, ' Chen Wang, ${ }^{2}$ Yujia Kong,' Liping Yang, ${ }^{3}$ Juan Li,' Gaopei Zhu,' Jing Guo, ${ }^{4}$ Qingfeng Zheng, ${ }^{5}$ Bo Zhang, (10 ${ }^{6}$ Suzhen Wang'

'Department of Health Statistics, School of Public Health, Weifang Medical University, Weifang, Shandong 261053, People's Republic of China; ${ }^{2}$ Department of Computer Science, Rutgers University, Piscataway, NJ 08854, USA; ${ }^{3}$ Center for Health and Medicine, Xijing Hospital, an Affiliated Hospital of Air Force Military Medical University, Xi'an, Shannxi 7 10032, People's Republic of China; ${ }^{4}$ Department of Health Policy and Management, School of Public Health, Peking University, Beijing 100191, People's Republic of China; ${ }^{5}$ Department of Thoracic Surgery, National Cancer Center/National Clinical Research Center for Cancer/Cancer Hospital, Chinese Academy of Medical Sciences and Peking Union Medical College, Beijing I0002I, People's Republic of China; ${ }^{6}$ Department of Neurology and ICCTR Biostatistics and Research Design Center, Boston Children's Hospital and Harvard Medical School, Boston, MA, 02II5, USA

Correspondence: Bo Zhang; Suzhen Wang

Email bo.zhang@childrens.harvard.edu; wangsz@wfmc.edu.cn
Objective: To evaluate the survival benefit of surgery and radiation for hepatocellular carcinoma (HCC) after adjusting for patient-specific and tumor-specific factors.

Methods: This study analyzed HCC patients who enrolled in the Surveillance, Epidemiology, and End Results (SEER) registry between January 2004 and December 2013. Of the 5552 HCC patients, 4597 received surgery and 955 received radiation. Patients who received radiation were further divided into 3 subgroups: 541 who received beam radiation (BR), 197 who received radioactive implants (RI), and 217 who received radioisotopes (RIT). Propensity score weighting analysis derived from generalized boosted models (GBMs) was performed to ensure well-balanced characteristics in all comparison groups.

Results: Overall survival rates and HCC-specific survival rates were higher in those receiving surgery compared with those receiving radiotherapy. This was confirmed by Cox proportional hazard regression both before and after inverse probability of treatment weighting (IPTW). Before IPTW, the RIT group had a better outcome than the BR group in terms of overall and HCC-specific survival rates, but there was no significant difference between the RI and BR groups. After IPTW, Cox proportional hazard regression demonstrated that both the RIT and RI groups had higher survival rates than the BR group.

Conclusion: In HCC patients, surgery was associated with higher survival rates compared with radiotherapy while adjusting for other factors. Among those who received radiotherapy, RIT and RI granted survival benefits.

Keywords: hepatocellular carcinoma, survival analysis, propensity score, generalized boosted models, inverse probability of treatment weighting, Cox proportional hazard models

\section{Introduction}

Hepatocellular carcinoma (HCC) is the third leading cause of cancer-related modality worldwide and the fifth most common malignant neoplasm. ${ }^{1,2}$ However, the overall prognosis for patients with $\mathrm{HCC}$ is unsatisfactory with a 5-year survival rate of less than $5 \%$, which is further reduced in patients who do not receive any liverspecific therapy. ${ }^{2}$ In recent years, many studies have identified locoregional treatment options specifically targeting intrahepatic lesions, including surgical resection, chemotherapy, and radiotherapy, as favorable factors affecting HCC prognosis and long-term survival. ${ }^{3,4}$ However, these studies have not taken into account the 
confounding socio-demographic and clinical predictors on the population level. In addition, there are currently no studies in the literature that specifically investigate the survival benefits of surgery and radiation therapy among HCC patients with adjustment for patient-specific and tumor-specific factors. There is also a lack of data further suggesting which type of radiation treatment is most effective if patients only receive radiation.

The purpose of this retrospective study was to use data from the Surveillance, Epidemiology, and End Results (SEER) registry to evaluate the survival benefits of surgery and radiotherapy in patients with HCC while adjusting for other potential confounding patient-specific and tumorspecific factors. To correct for selection bias and other potential confounders, we adopted the approach of inverse probability of treatment weighting (IPTW) ${ }^{5-7}$ as an alternative to conventional survival analysis methods. In addition to analyzing the original SEER sample, this approach created a so-called "pseudo-sample" to estimate a weighted effect on the time-to-event outcomes of both overall survival and HCC-specific survival. In the pseudo-sample, patients from the original sample were assigned a weight derived from the IPTW. An IPTW Kaplan-Meier estimator was used to estimate survival curves, and IPTW Cox proportional hazard models were fit to the SEER data to estimate hazard ratios. ${ }^{8-10}$ SEER registry patients who received radiotherapy were further divided into 3 subgroups according to the type of radiation therapy they received: beam radiation, radioactive implants, or radioisotopes. Our study evaluated and compared the survival benefit of each type of radiation therapies among these patients.

\section{Methods}

\section{Data}

The SEER database, hosted by the National Cancer Institute in the National Institutes of Health, is the largest publicly available cancer dataset in the United States and provides cancer incidence, treatment, and survival data from population-based cancer registries. Specifically, the SEER 9 registry database (1975-2013) was the data source for this study. Patients with pathologically confirmed HCC lesions who enrolled in the registry between 2004 to 2013 were identified by ICD-O-3 histology codes $8170 / 3-8175 / 3$, combined with the liver site codes C22.0. ${ }^{9}$ A total of 18,514 HHC patients were identified, then limited to those who only received surgery or radiation treatment. Exclusion criteria included (i) diagnosis at autopsy; (ii) diagnosis by death certificates only; (iii) surgery not recommended; (iv) age $<20$ years; (v) T0 stage, or unknown T, N, or M stage; and (vi) radiation therapy other than beam radiation, radioactive implants, or radioisotopes. Finally, a total number of 5552 patients were included in our study, consisting of 4597 who received surgery and 955 who received radiation therapy. Of the 955 patients who received radiation therapy, 541 received beam radiation (BR), 197 received radioactive implants (RI), and 217 received radioisotopes (RIT).

This study was approved by the Ethics Review Board of Weifang Medical University and conformed to the provisions of the Declaration of Helsinki.

\section{Statistical Analysis}

Propensity score weighting analysis derived from generalized boosted models (GBMs) has been approved as an effective analysis approach to reduce baseline bias and to compare long-term survival in different groups. ${ }^{10-13}$ Therefore, we adopted it as our primary analysis approach to assess and compare the survival rates of HCC patients who received either surgery or radiation treatment. The covariates potentially associated with treatment selection, including age, gender, race, marital status, tumor size, tumor grade, $\mathrm{T}$ stage, $\mathrm{M}$ stage, $\mathrm{N}$ stage, and disease extent condition, were included in the generation of propensity scores. GBMs with these covariates were used to generate a continuous propensity score and estimate the probability that a patient would undergo surgery or radiation; then, propensity score weighting analysis was conducted with these generated propensity scores. The absolute standardized mean difference (ASMD) or the KolmogorovSmirnov (KS) statistic was taken as the stopping rule for the complexity of the GBM. ${ }^{10,14}$ ASMD values greater than 0.2 were considered to be indicative of moderate imbalance while KS values greater than 0.10 were considered to be indicative of imbalance. The ASMD and the KS distances were used to measure balance between the different groups for each pretreatment covariate.

Baseline characteristics of the different treatment groups were compared and evaluated by the chi-square test or the Mantel-trend test for categorical variables in the original sample. To minimize the impact of selection bias and other potential confounders, a pseudo-sample was created by rigorous adjustment using IPTW of the propensity scores. ${ }^{15,16}$ The propensity scores were estimated by the GBMs to predict the probability of a patient undergoing each treatment. The following methods were 
employed to estimate overall survival rates: IPTW KaplanMeier estimator for estimating survival curves and IPTW Cox proportional hazard models for estimating hazard ratios. ${ }^{17-19}$ All significance tests were two-tailed, and $\mathrm{P}$ values less than 0.05 were considered statistically significant. All statistical analyses were conducted in the R software environment (version 3.5.0). The GBM propensity score weights were obtained using the Toolkit for Weighting and Analysis of Nonequivalent Groups package. ${ }^{14} \mathrm{R}$ package "coxphw" was used to conduct IPTW Cox proportional hazard regression. ${ }^{19,20}$

\section{Results}

\section{Demographic and Clinical Characteristics of the Original Sample and the Pseudo-}

\section{Sample at Baseline}

Among the 5552 HCC patients included in this study, the median (25\% quantile and $75 \%$ quantile) follow-up periods were 21.0 (8.0 and 45.0), 26.0 (11.0 and 51.0), and 7.0 (3.0 and 15.0) months in the entire sample, surgery, and radiation groups, respectively. The mean (standard deviation) ages of the entire sample, surgery, and radiation groups were 61.76 (10.78), 63.56 (11.01), and 61.39 (10.69) years, respectively. Among the 955 radiation patients, the median (25\% quantile and $75 \%$ quantile) follow-up period was 5.0 (2.0 and 12.0), 8.0 (4.0 and 15.0), and 9.0 (5.0 and 20.0) months in the BR, RI, and RIT groups, respectively.

Baseline demographic and clinical characteristics of the HCC patients are listed in Table 1. Before IPTW analysis in the original sample, the surgery and radiation groups took significantly different proportions in the categories of all covariates, except for marital status ( $\mathrm{P}=$ 0.193). However, after IPTW balancing in the pseudosample, the two groups took similar proportions (no significant difference was detected in proportions) in the categories of all covariates (Table 1).

Baseline characteristics of the 955 patients in the radiation group are summarized in Table 2. Before IPTW analysis in the original sample, the 3 radiation groups had similar proportions in the categories of age, gender, race, and marital status. However, a significant difference was recognized for tumor size $(\mathrm{P}=0.002)$, tumor grade ( $\mathrm{P}$ $=0.031)$, T stage $(\mathrm{P}<0.001), \mathrm{N}$ stage $(\mathrm{P}<0.001)$, M stage $(\mathrm{P}<0.001)$, and disease extent condition $(\mathrm{P}<0.001)$. After IPTW balancing in the pseudo-sample, the 3 radiation treatment groups took the similar proportion in the categories of all covariates (Table 2).

\section{Survival in the Original Sample}

Figure 1 shows both the unadjusted (without propensity score IPTW) and adjusted (with propensity score IPTW) estimated 5-year Kaplan-Meier overall survival curves and HCC-specific survival curves between the surgery and radiation groups. Table 3 shows the analysis results derived from Cox proportional hazard regression (hazard ratios, their $95 \%$ confidence intervals or CIs, and $\mathrm{P}$ values) for all-cause mortality and $\mathrm{HCC}$-specific mortality in the original sample; Table 4 displays these results in the pseudo-sample.

In the original sample, the mortality rates were $45.7 \%$ in the surgery group (2100 patients died during the study period) and $71.0 \%$ in the radiation group (678 patients died). The overall survival rates at the end of the $1^{\text {st }}, 3^{\text {rd }}$, and $5^{\text {th }}$ years were $82.6 \%, 58.4 \%$ and $44.7 \%$, respectively, in the surgery group, and were $37.9 \%, 15.0 \%$, and $7.7 \%$, respectively, in the radiation group (Figure 1A). Corresponding median survival times were 51 months in the surgery group and 9 months in the radiation group. The results obtained from the Cox proportional hazard regression revealed that the patients who received surgery gained significant benefits in terms of survival rates, compared with the patients who received radiation (all-cause mortality hazard ratio, 0.48 ; CI, 0.430.54). While HCC-specific survival rates at the $1^{\text {st }}, 3^{\text {rd }}$, and $5^{\text {th }}$ years were $88.5 \%, 71.1 \%$, and $60.6 \%$, respectively, in the surgery group, they were $47.5 \%, 24.4 \%$, and $15.0 \%$, respectively, in the radiation group (Figure 1B). Corresponding HCC-specific median survival times were 105 months in the surgery group and 11 months in the radiation group. Analysis results from Cox proportional hazard regression revealed that the HCC-specific survival rate in the surgery group was still superior to that in the radiation group (HCC-specific mortality hazard ratio, 0.44; CI 0.38-0.50; $\mathrm{P}<0.001)$.

The following covariates were risk factors for HCC-specific mortality: age 40 to 59 years (versus age 20-39 years; hazard ratio, 1.50; CI, 1.09-2.05; P = 0.012), age 60 to 79 years (versus age 20-39 years; hazard ratio, 1.49; CI, 1.082.04; $\mathrm{P}=0$ 0.014), male (versus female; hazard ratio, 1.14; CI, 1.10-1.40; $\mathrm{P}<0.001$ ), black race (versus white; hazard ratio, 1.16; $\mathrm{CI}, 1.01-1.32 ; \mathrm{P}=0.032$ ), single (never married) social status (versus married social status; hazard ratio, 1.32; CI, 1.16-1.50; $\mathrm{P}<0.001$ ), separated social status (versus married social status; hazard ratio, 1.90; CI, 1.34-2.70; $\mathrm{P}<0.001$ ), divorced social status (versus married social 
Table I Baseline Demographic and Clinical Characteristics of Patients Before and After Inverse Probability of Treatment Weighting (IPTW)

\begin{tabular}{|c|c|c|c|c|c|c|}
\hline \multirow[t]{2}{*}{ Variable } & \multicolumn{2}{|l|}{ Before IPTW $(n=5552)$} & \multirow[t]{2}{*}{$P$ value } & \multicolumn{2}{|l|}{ After IPTW } & \multirow[t]{2}{*}{ Minimum $P$ value } \\
\hline & Surgery $(n=4597, \%)$ & Radiation ( $n=955, \%)$ & & Surgery (\%) & Radiation (\%) & \\
\hline $\begin{array}{l}\text { Age group } \\
\begin{array}{l}20-39 \\
40-59 \\
60-79 \\
\geq 80\end{array}\end{array}$ & $\begin{array}{l}106(2.3) \\
2021(44.0) \\
2225(48.4) \\
245(5.3)\end{array}$ & $\begin{array}{l}15(1.6) \\
359(37.6) \\
490(51.3) \\
91(9.5)\end{array}$ & $<0.001$ & $\begin{array}{l}(2.1) \\
(43.2) \\
(48.9) \\
(5.7)\end{array}$ & $\begin{array}{l}(0.6) \\
(42.6) \\
(50.7) \\
(6.1)\end{array}$ & 0.078 \\
\hline $\begin{array}{c}\text { Gender } \\
\text { Female } \\
\text { Male }\end{array}$ & $\begin{array}{l}\text { II } 25(24.5) \\
3472(75.5)\end{array}$ & $\begin{array}{l}176(18.4) \\
779(81.6)\end{array}$ & $<0.001$ & $\begin{array}{l}(23.4) \\
(76.6)\end{array}$ & $\begin{array}{l}(20.0) \\
(80.0)\end{array}$ & 0.163 \\
\hline $\begin{array}{l}\text { Race } \\
\text { White } \\
\text { Black } \\
\text { Chinese } \\
\text { Others }\end{array}$ & $\begin{array}{l}2880(62.6) \\
575(12.5) \\
351(7.6) \\
791(17.2)\end{array}$ & $\begin{array}{l}648(67.9) \\
166(17.4) \\
19(2.0) \\
122(12.8)\end{array}$ & $<0.001$ & $\begin{array}{l}(62.8) \\
(14.1) \\
(6.7) \\
(16.4)\end{array}$ & $\begin{array}{l}(67.2) \\
(14.8) \\
(5.4) \\
(12.6)\end{array}$ & 0.371 \\
\hline $\begin{array}{l}\text { Marital status } \\
\text { Married/domestic partner } \\
\text { Single (never married) } \\
\text { Separated } \\
\text { Divorced } \\
\text { Widowed } \\
\text { Other/unknown }\end{array}$ & $\begin{array}{l}2660(57.9) \\
790(17.2) \\
64(1.4) \\
552(12.0) \\
348(7.6) \\
183(4.0)\end{array}$ & $\begin{array}{l}549(57.5) \\
176(18.4) \\
7(0.7) \\
127(13.3) \\
69(7.2) \\
27(2.8)\end{array}$ & 0.193 & $\begin{array}{l}(58.9) \\
(17.2) \\
(1.2) \\
(11.7) \\
(7.2) \\
(3.7)\end{array}$ & $\begin{array}{l}(60.5) \\
(18.2) \\
(0.7) \\
(11.5) \\
(5.4) \\
(3.8)\end{array}$ & 0.662 \\
\hline $\begin{array}{l}\text { Tumor size }(\mathrm{cm}) \\
\quad<3.0 \\
3.0-4.9 \\
5.0-10.0 \\
>10.0 \\
\text { unknown }\end{array}$ & $\begin{array}{l}1936(42.1) \\
1289(28.0) \\
78 \mid(17.0) \\
374(8.1) \\
217(4.7)\end{array}$ & $\begin{array}{l}157(16.4) \\
183(19.2) \\
296(31.0) \\
143(15.0) \\
176(18.4)\end{array}$ & $<0.001$ & $\begin{array}{l}(37.9) \\
(26.5) \\
(19.2) \\
(9.4) \\
(7.1)\end{array}$ & $\begin{array}{l}(34.7) \\
(26.1) \\
(22.6) \\
(9.4) \\
(7.2)\end{array}$ & 0.629 \\
\hline $\begin{array}{l}\text { Grade } \\
\text { Grade I } \\
\text { Grade II } \\
\text { Grade III } \\
\text { Grade IV } \\
\text { Unknown grade }\end{array}$ & $\begin{array}{l}797(17.3) \\
1458(31.7) \\
467(10.2) \\
39(0.8) \\
1836(39.9)\end{array}$ & $\begin{array}{l}88(9.2) \\
126(13.2) \\
68(7.1) \\
1(0.1) \\
672(70.4)\end{array}$ & $<0.001$ & $\begin{array}{l}(15.8) \\
(28.6) \\
(9.7) \\
(0.7) \\
(45.3)\end{array}$ & $\begin{array}{l}(14.0) \\
(23.2) \\
(9.9) \\
(0.0) \\
(52.9)\end{array}$ & 0.060 \\
\hline $\begin{array}{l}\text { T stage } \\
\text { T1 } \\
\text { T2 } \\
\text { T3 } \\
\text { T4 } \\
\text { TX }\end{array}$ & $\begin{array}{l}2664(58.0) \\
1303(28.3) \\
404(8.8) \\
94(2.0) \\
132(2.9)\end{array}$ & $\begin{array}{l}292(30.6) \\
180(18.8) \\
316(33.1) \\
44(4.6) \\
123(12.9)\end{array}$ & $<0.001$ & $\begin{array}{l}(53.2) \\
(26.8) \\
(13.6) \\
(2.3) \\
(4.1)\end{array}$ & $\begin{array}{l}(51.9) \\
(26.2) \\
(14.4) \\
(2.9) \\
(4.5)\end{array}$ & 0.902 \\
\hline $\begin{array}{l}\text { N stage } \\
\text { No } \\
\text { NI } \\
\text { NX }\end{array}$ & $\begin{array}{l}4325(94.1) \\
78(1.7) \\
194(4.2)\end{array}$ & $\begin{array}{l}720(75.4) \\
97(10.2) \\
138(14.5)\end{array}$ & $<0.001$ & $\begin{array}{l}(90.9) \\
(3.0) \\
(6.2)\end{array}$ & $\begin{array}{l}(90.4) \\
(3.4) \\
(6.2)\end{array}$ & 0.849 \\
\hline $\begin{array}{c}\text { M stage } \\
\text { MO } \\
\text { MI }\end{array}$ & $\begin{array}{l}4422(96.2) \\
85(1.8)\end{array}$ & $\begin{array}{l}581(60.8) \\
346(36.2)\end{array}$ & $<0.001$ & $\begin{array}{l}(90.8) \\
(7.2)\end{array}$ & $\begin{array}{l}(89.3) \\
(8.9)\end{array}$ & 0.488 \\
\hline
\end{tabular}

(Continued) 
Table I (Continued).

\begin{tabular}{|c|l|l|l|l|l|l|}
\hline \multirow{2}{*}{ Variable } & \multicolumn{2}{|l|}{ Before IPTW (n=5552) } & \multirow{2}{*}{ P value } & \multicolumn{2}{l|}{ After IPTW } & \multirow{2}{*}{ Minimum P value } \\
\cline { 2 - 3 } & Surgery (n=4597, \%) & Radiation (n=955, \%) & & Surgery (\%) & Radiation (\%) & \\
\hline MX & $90(2.0)$ & $28(2.9)$ & & $(2.0)$ & $(1.8)$ & \\
\hline Disease extent condition & & & $<0.001$ & & & \\
Localized & $3594(78.2)$ & $311(32.6)$ & & $(70.9)$ & $(67.9)$ & \\
Regional & $824(17.9)$ & $279(29.2)$ & $(19.8)$ & $(21.6)$ & \\
Distant & $108(2.3)$ & $350(36.6)$ & $(7.7)$ & $(9.3)$ & \\
Unstaged & $71(1.5)$ & $15(1.6)$ & $(1.6)$ & $(1.2)$ & \\
\hline
\end{tabular}

status; hazard ratio, 1.32; CI, 1.14-1.53; P <0.001), widowed social status (versus married social status; hazard ratio, 1.51; CI, 1.26-1.83; $\mathrm{P}<0.001$ ), tumor size level 3.0 to $4.9 \mathrm{~cm}$ (versus <3.0 cm; hazard ratio, 1.47; CI, 1.29-1.69; $\mathrm{P}<$ 0.001 ), tumor size level 5.0 to $10.0 \mathrm{~cm}$ (versus $<3.0 \mathrm{~cm}$; hazard ratio, 1.57; $\mathrm{CI}, 1.35-1.83 ; \mathrm{P}<0.001)$, tumor size level $>10.0 \mathrm{~cm}$ (versus $<3.0 \mathrm{~cm}$; hazard ratio, 2.26; CI, 1.90-2.69; $\mathrm{P}<0.001$ ), grade III (versus grade I; hazard ratio, 1.78; CI, 1.47-2.16; $\mathrm{P}<0.001$ ), T2 (versus T1; hazard ratio, 1.14; CI, 1.01-1.29; $\mathrm{P}=0.039$ ), T3 (versus T1; hazard ratio, 1.70; CI, 1.44-2.00; $\mathrm{P}<0.001$ ), $\mathrm{M} 1$ (versus $\mathrm{M} 0$; hazard ratio, 1.63; CI, 1.06-2.50; $\mathrm{P}=0.026)$, regional disease extent (versus localized status; hazard ratio, 1.41; CI, 1.24-1.62; P < 0.001 ), and distant disease extent (versus localized status; hazard ratio, 1.91; $\mathrm{CI}, 1.24-2.96 ; \mathrm{P}=0$ 0.003).

\section{Survival in the Pseudo-Sample}

After IPTW, the patients in the pseudo-sample were wellbalanced across the surgery and radiation groups (Table 1). The overall survival rates in the surgery group at the $1^{\text {st }}$, $3^{\text {rd }}$, and $5^{\text {th }}$ years were $78.2 \%, 53.4 \%$, and $40.0 \%$, respectively, and were $56.0 \%, 28.0 \%$, and $15.8 \%$, respectively, in the radiation group (Figure 1A). Corresponding median survival times were 43 months in the surgery group and 17 months in the radiation group. Analysis results obtained from the IPTW Cox proportional hazard regression demonstrated that the surgery group was superior to the radiation group in terms of all-cause mortality rates in the pseudo-sample (Figure 1B, Table 4). The HCC-specific survival rates at the $1^{\text {st }}, 3^{\text {rd }}$, and $5^{\text {th }}$ years were $84.2 \%$, $65.7 \%$, and $55.1 \%$, respectively, in the surgery group, and were $65.9 \%, 38.2 \%$, and $26.0 \%$, respectively, in the radiation group. Corresponding HCC-specific median survival times were 83 months in the surgery group and 24 months in the radiation group. The IPTW Cox proportional hazard regression showed a significant difference in HCC-specific mortality rates between the two groups (Table 4).

We discovered that the following covariates were risk factors for HCC-specific mortality: age 40-59 years (hazard ratio, 1.53; CI, 1.06-2.21; $\mathrm{P}=0.024$ ), single (never married) social status (versus married social status; hazard ratio, 1.35; $\mathrm{CI}, 1.05-1.73 ; \mathrm{P}=0.021)$, separated social status (versus married social status; hazard ratio, 1.74; CI, 1.03-2.94; $\mathrm{P}=0.039$ ), divorced social status (versus married social status; hazard ratio, 1.32; CI, 1.05-1.65; $\mathrm{P}=0.018$ ), widowed social status (versus married social status; hazard ratio, $1.48 ; \mathrm{CI}, 1.14-1.92 ; \mathrm{P}=$ 0.003 ), grade III (versus grade I; hazard ratio, 1.81; CI, 1.18-2.76; $\mathrm{P}=0.006$ ), T3 (versus $\mathrm{T} 1$; hazard ratio, 1.70; CI, 1.29-2.24; $\mathrm{P}<0.001$ ), and T4 (versus T1; hazard ratio, 2.13; CI, 1.05-4.32; $\mathrm{P}=0.035)$. In addition, tumor size level was significantly associated with an increased HCCspecific mortality (Table 4).

\section{Survival in the 3 Radiation Subgroups}

Figure 2 shows both the unadjusted (without propensity score IPTW) and adjusted (with propensity score IPTW) estimated 5-year Kaplan-Meier HCC-specific survival curves among the 3 radiation groups. Table 5 shows the analysis results derived from Cox proportional hazard regression (hazard ratios, their 95\% CIs and $\mathrm{P}$ values) for all-cause mortality and $\mathrm{HCC}$-specific mortality in the 3 original radiation subgroups; Table 6 displays these results in the pseudo-sample subgroups.

In the 3 original sample radiation subgroups, the mortality rates were $81.1 \%$ in the BR group (439 patients died during the study period), $59.9 \%$ in the RI group (118 patients died), and $55.8 .0 \%$ in the RIT group (121 patients died). The overall survival rates at the end of the $1^{\text {st }}, 3^{\text {rd }}$, and $5^{\text {th }}$ years were $27.9 \%, 10.4 \%$, and $3.5 \%$, respectively, in the BR group; $46.0 \%, 14.8 \%$, and $4.9 \%$, respectively, in 
Table 2 Clinical Characteristics of Radiation Patients Before and After Inverse Probability of Treatment Weighting (IPTW)

\begin{tabular}{|c|c|c|c|c|c|c|c|c|}
\hline \multirow[t]{2}{*}{ Variable } & \multicolumn{3}{|c|}{ Before IPTW $(n=955)$} & \multirow[b]{2}{*}{$P$ value } & \multicolumn{4}{|c|}{ After IPTW } \\
\hline & BR $(n=541, \%)$ & RI $(n=197, \%)$ & $\operatorname{RIT}(n=217, \%)$ & & BR (\%) & RI (\%) & RIT (\%) & Minimum $P$ value \\
\hline Age & & & & 0.413 & & & & 0.384 \\
\hline $20-39$ & II (2.0) & $2(1.0)$ & $2(0.9)$ & & $(1.6)$ & $(1.5)$ & $(1.0)$ & \\
\hline $40-59$ & 210 & $64(32.5)$ & $85(39.2)$ & & $(37.5)$ & $(33.9)$ & $(33.2)$ & \\
\hline $60-79$ & $265(49.0)$ & $112(56.9)$ & $113(52.1)$ & & $(51.8)$ & $(56.8)$ & (59.7) & \\
\hline$\geq 80$ & $55(10.2)$ & $19(9.6)$ & $17(7.8)$ & & $(9.2)$ & $(7.8)$ & $(6.0)$ & \\
\hline Gender & & & & 0.338 & & & & 0.161 \\
\hline Female & $91(16.8)$ & $41(20.8)$ & $44(20.3)$ & & $(18.8)$ & $(21.8)$ & $(15.3)$ & \\
\hline Male & $450(83.2)$ & $156(79.2)$ & $173(79.3)$ & & $(81.2)$ & $(78.2)$ & (84.7) & \\
\hline Race & & & & 0.151 & & & & 0.118 \\
\hline White & $362(66.9)$ & $139(70.6)$ & $147(67.7)$ & & (69.8) & $(71.4)$ & (78.1) & \\
\hline Black & $95(17.6)$ & $36(18.3)$ & $35(16.1)$ & & $(16.7)$ & $(15.7)$ & $(11.5)$ & \\
\hline Chinese & $9(1.7)$ & $\mathrm{I}(0.5)$ & $9(4.1)$ & & $(1.3)$ & $(1.0)$ & $(1.5)$ & \\
\hline Others & $75(13.9)$ & $21(10.7)$ & $26(12.0)$ & & $(12.2)$ & $(11.9)$ & $(8.9)$ & \\
\hline Marital status & & & & 0.378 & & & & 0.513 \\
\hline Married/domestic partner & $298(55.1)$ & $114(57.9)$ & $137(63.1)$ & & (58.6) & $(63.0)$ & $(67.1)$ & \\
\hline Single (never married) & $109(20.1)$ & $36(18.3)$ & $31(14.3)$ & & $(18.3)$ & $(16.2)$ & (16.9) & \\
\hline Separated & $5(0.9)$ & $\mathrm{I}(0.5)$ & $\mathrm{I}(0.5)$ & & $(0.8)$ & $(0.3)$ & $(0.3)$ & \\
\hline Divorced & $73(13.5)$ & $22(11.2)$ & $32(14.7)$ & & (12.8) & $(11.6)$ & $(9.9)$ & \\
\hline Widowed & $39(7.2)$ & $16(8.1)$ & $14(6.5)$ & & $(7.3)$ & $(7.2)$ & $(4.6)$ & \\
\hline Other/unknown & $17(3.1)$ & $8(4.1)$ & $2(0.9)$ & & (2.2) & (I.6) & (I.I) & \\
\hline Tumor size $(\mathrm{cm})$ & & & & 0.002 & & & & 0.857 \\
\hline$<3$ & $89(16.5)$ & $34(17.3)$ & $34(15.7)$ & & $(16.2)$ & $(16.5)$ & $(14.2)$ & \\
\hline $3-4.9$ & $94(17.4)$ & $40(20.3)$ & $49(22.6)$ & & $(19.4)$ & $(19.4)$ & (22.5) & \\
\hline $5-10$ & $150(27.7)$ & $68(34.5)$ & 78 (35.9) & & (30.8) & $(30.0)$ & (34.8) & \\
\hline$>10$ & $81(15.0)$ & $29(14.7)$ & $33(15.2)$ & & $(15.1)$ & $(15.3)$ & (13.7) & \\
\hline Unknown & $127(23.5)$ & $26(13.2)$ & $23(10.6)$ & & (18.5) & $(18.7)$ & (14.9) & \\
\hline Grade & & & & 0.031 & & & & 0.872 \\
\hline Grade I & $37(6.8)$ & $26(13.2)$ & $25(11.5)$ & & (8.2) & $(9.9)$ & $(10.1)$ & \\
\hline Grade II & $65(12.0)$ & $30(15.2)$ & $31(14.3)$ & & $(13.1)$ & $(13.1)$ & (12.9) & \\
\hline Grade III/IV & $47(8.7)$ & $10(5.1)$ & $12(5.5)$ & & (7.4) & (6.8) & (9.5) & \\
\hline Unknown Grade & $392(72.5)$ & $13 \mid(66.5)$ & $149(68.7)$ & & (71.4) & $(70.1)$ & $(67.5)$ & \\
\hline T stage & & & & $<0.001$ & & & & 0.476 \\
\hline $\mathrm{TI}$ & $183(33.8)$ & $50(25.4)$ & $59(27.2)$ & & (30.4) & $(27.4)$ & (24.5) & \\
\hline $\mathrm{T} 2$ & $76(14.0)$ & $45(22.8)$ & $59(27.2)$ & & (17.9) & $(18.8)$ & $(17.5)$ & \\
\hline T3 & $154(28.5)$ & $82(41.6)$ & $80(36.9)$ & & (33.9) & $(37.5)$ & $(43.6)$ & \\
\hline $\mathrm{T} 4$ & $30(5.5)$ & $7(3.6)$ & $7(3.2)$ & & (4.6) & (3.8) & (2.9) & \\
\hline TX (99) & $98(18.1)$ & $13(6.6)$ & $12(5.5)$ & & $(13.2)$ & $(12.5)$ & (11.5) & \\
\hline $\mathrm{N}$ stage & & & & $<0.001$ & & & & 0.866 \\
\hline No & $368(68.0)$ & $170(86.3)$ & $182(83.9)$ & & $(75.1)$ & $(77.6)$ & (73.1) & \\
\hline $\mathrm{NI}$ & 64 (11.8) & $16(8.1)$ & $17(7.8)$ & & $(10.2)$ & $(9.8)$ & (II.6) & \\
\hline$N X$ & $109(20.1)$ & II (5.6) & $18(8.3)$ & & $(14.6)$ & $(12.7)$ & (15.3) & \\
\hline M stage & & & & $<0.001$ & & & & 0.314 \\
\hline Mo & $210(38.8)$ & $17 \mid(86.8)$ & $200(92.2)$ & & $(59.0)$ & $(68.2)$ & (66.7) & \\
\hline MI & $320(59.1)$ & $18(9.1)$ & $8(3.7)$ & & (38.2) & $(28.6)$ & (30.3) & \\
\hline$M X$ & II (2.0) & $8(4.1)$ & $9(4.1)$ & & (2.8) & (3.2) & (3.0) & \\
\hline $\begin{array}{l}\text { Disease extent condition } \\
\text { Localized }\end{array}$ & $132(24.4)$ & $85(43.1)$ & $94(43.3)$ & $<0.001$ & (31.8) & $(37.1)$ & (33.7) & 0.345 \\
\hline
\end{tabular}

(Continued) 
Table 2 (Continued).

\begin{tabular}{|l|l|l|l|l|l|l|l|l|}
\hline \multirow{2}{*}{ Variable } & \multicolumn{2}{|l|}{ Before IPTW (n = 955) } & \multicolumn{3}{l|}{ After IPTW } \\
\cline { 2 - 9 } & BR (n= 54I, \%) & RI (n= 197, \%) & RIT (n= 217, \%) & P value & BR (\%) & RI (\%) & RIT (\%) & Minimum P value \\
\hline Regional & $78(14.4)$ & $91(46.2)$ & $110(50.7)$ & & $(28.1)$ & $(33.0)$ & $(33.5)$ & \\
Distant & $323(59.7)$ & $17(8.6)$ & $10(4.6)$ & & $(38.6)$ & $(28.7)$ & $(31.3)$ & $(1.6)$ \\
Unstaged & $8(1.5)$ & $4(2.0)$ & $3(1.4)$ & & $(1.5)$ & $(1.3)$ & $(1.6)$ \\
\hline
\end{tabular}

Abbreviations: BR, beam radiation; RI, radioactive implants; RIT, radioisotopes.

the RI group; and $52.6 \%, 25.8 \%$, and $12.0 \%$, respectively, in the RIT group. Corresponding median survival times were 6.11, and 14 months in the BR, RI, and RIT groups, respectively. The results obtained from the Cox proportional hazard regression revealed that the patients who received RIT gained significant benefits in terms of survival rates, compared with the patients who received BR (all-cause mortality hazard ratio, 0.68; CI 0.54-0.87; $\mathrm{P}=0.002$ ); however, there were no significant superior benefits among patients who received RI compared with those who received BR (all-cause mortality hazard ratio, 0.86; CI 0.68-1.08; $\mathrm{P}=0.191$ ). The HCC-specific survival rates at the $1^{\text {st }}, 3^{\text {rd }}$, and $5^{\text {th }}$ years were $36.6 \%, 17.5 \%$, and $10.2 \%$, respectively, in the BR group; $58.3 \%, 26.7 \%$, and $10.7 \%$, respectively, in the RI group; and $61.1 \%, 38.4 \%$, and $35.6 \%$, respectively, in the RIT group (Figure 2A). Corresponding $\mathrm{HCC}$-specific median survival times were 8.17, and 23 months in the BR, RI, and RIT groups, respectively. Analysis results from $\mathrm{Cox}$ proportional hazard regression revealed that the $\mathrm{HCC}$-specific survival rate in the RIT group was still superior to that in the BR group (HCC-specific mortality hazard ratio, 0.69; CI 0.52 $0.91 ; \mathrm{P}=0.008$ ), and there were no significant benefits to HCC-specific survival rate in the RI group compared with the BR group (HCC-specific mortality hazard ratio, 0.85; CI $0.65-1.11 ; \mathrm{P}=0.227)$.

The following covariates were risk factors for HCCspecific mortality: tumor size level 3.0 to $4.9 \mathrm{~cm}$ (versus $<3.0 \mathrm{~cm}$; hazard ratio, 1.68; CI, 1.19-2.37; $\mathrm{P}=0.003$ ), tumor size level 5.0 to $10.0 \mathrm{~cm}$ (versus $<3.0 \mathrm{~cm}$; hazard ratio, 1.69; CI, 1.19-2.39; $\mathrm{P}=0.003)$, tumor size level $>10.0 \mathrm{~cm}$ (versus $<3.0 \mathrm{~cm}$; hazard ratio, 1.95 ; CI, 1.31 2.90; $\mathrm{P}=0.001$ ), grade III (versus grade I; hazard ratio, 1.58; CI, 1.03-2.45; $\mathrm{P}=0.038$ ), T3 (versus T1; hazard ratio, 1.56; CI, 1.18-2.06; $\mathrm{P}=0.002)$, and distant disease extent (versus localized status; hazard ratio, 3.28; CI, 1.35-7.95; $\mathrm{P}=0.009$ ).

After IPTW, the patients in the pseudo-sample were well-balanced across the 3 radiation groups (Table 2).
Analysis results obtained from the IPTW Cox proportional hazard regression demonstrated that the RI and RIT groups were both superior to the BR group in terms of all-cause mortality rates in the pseudo-sample (Table 6). HCC-specific survival rates at the $1^{\text {st }}, 3^{\text {rd }}$, and $5^{\text {th }}$ years were $42.8 \%$, $22.4 \%$, and $7.8 \%$, respectively, in the BR group; $47.5 \%$, $21.6 \%$, and $12.0 \%$, respectively, in the RI group; and $52.9 \%, 33.5 \%$, and $31.4 \%$, respectively, in the RIT group. Corresponding HCC-specific median survival times were 10, 12, and 17 months in the BR, RI, and RIT groups, respectively. The IPTW Cox proportional hazard regression showed that the RIT and RI groups both outperformed the BR group in terms of HCC-specific mortality rates in the pseudo-sample (Figure 2B, Table 6).

We also discovered that the following covariates were risk factors for HCC-specific mortality: male (versus female; hazard ratio, 1.37; $\mathrm{CI}, 1.15-1.64 ; \mathrm{P}=0.001)$, tumor size level 3.0 to $4.9 \mathrm{~cm}$ (versus $<3.0 \mathrm{~cm}$; hazard ratio, 1.83; CI, 1.46-2.29; $\mathrm{P}<0.001$ ), tumor size level 5.0 to $10.0 \mathrm{~cm}$ (versus $<3.0 \mathrm{~cm}$; hazard ratio, 1.40 ; $\mathrm{CI}, 1.11-$ 1.77; $\mathrm{P}=0.004$ ), tumor size level $>10.0 \mathrm{~cm}$ (versus $<3.0 \mathrm{~cm}$; hazard ratio, 1.97; CI, 1.52-2.56; $\mathrm{P}<0.001)$, T2 (versus T1; hazard ratio, 1.31; CI, 1.10-1.65; $\mathrm{P}=0.004$ ), T3 (versus T1; hazard ratio, 2.19; CI, 1.81-2.64; $\mathrm{P}<$ 0.001 ), N1 (versus N0; hazard ratio, 0.62; CI, 0.50-0.77; $\mathrm{P}<0.001$ ), and distant disease extent (versus localized status; hazard ratio, 2.12; CI, 1.21-3.72; $\mathrm{P}=0$ 0.008). The male risk factor was not identified in the unadjusted Cox proportional hazard regression.

\section{Discussion}

Surgical resection and liver transplantation are the main curative treatments for HCC. Unfortunately, only $20 \%$ to $30 \%$ of HCC patients, mostly diagnosed by regular screening, may benefit from these therapies. ${ }^{21,22}$ While surgical resection remains the primary treatment approach to $\mathrm{HCC}$, it is not always feasible due to patient comorbidities or tumor characteristics. In this circumstance, radiotherapy becomes an alternative. Tumor location is a factor 


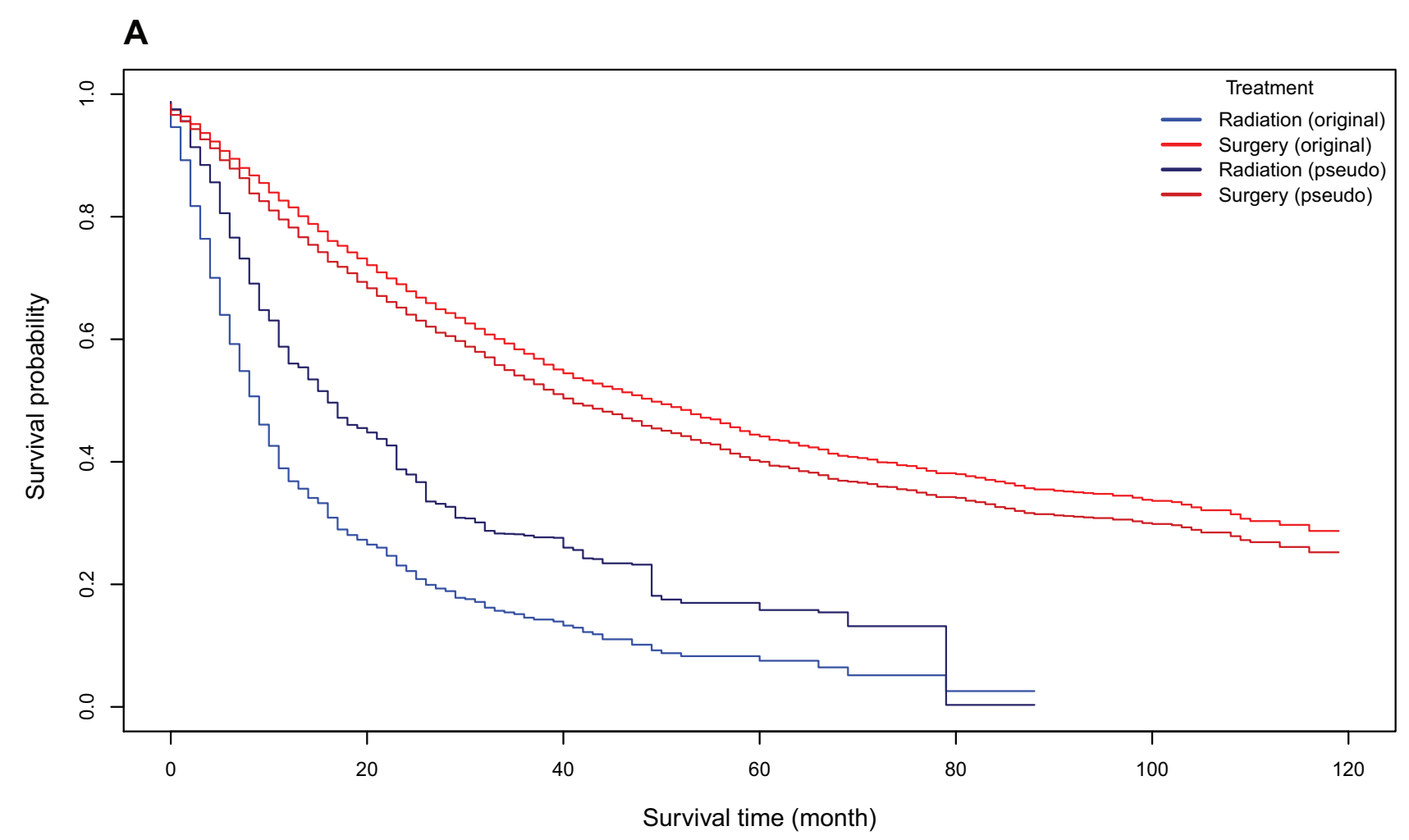

B

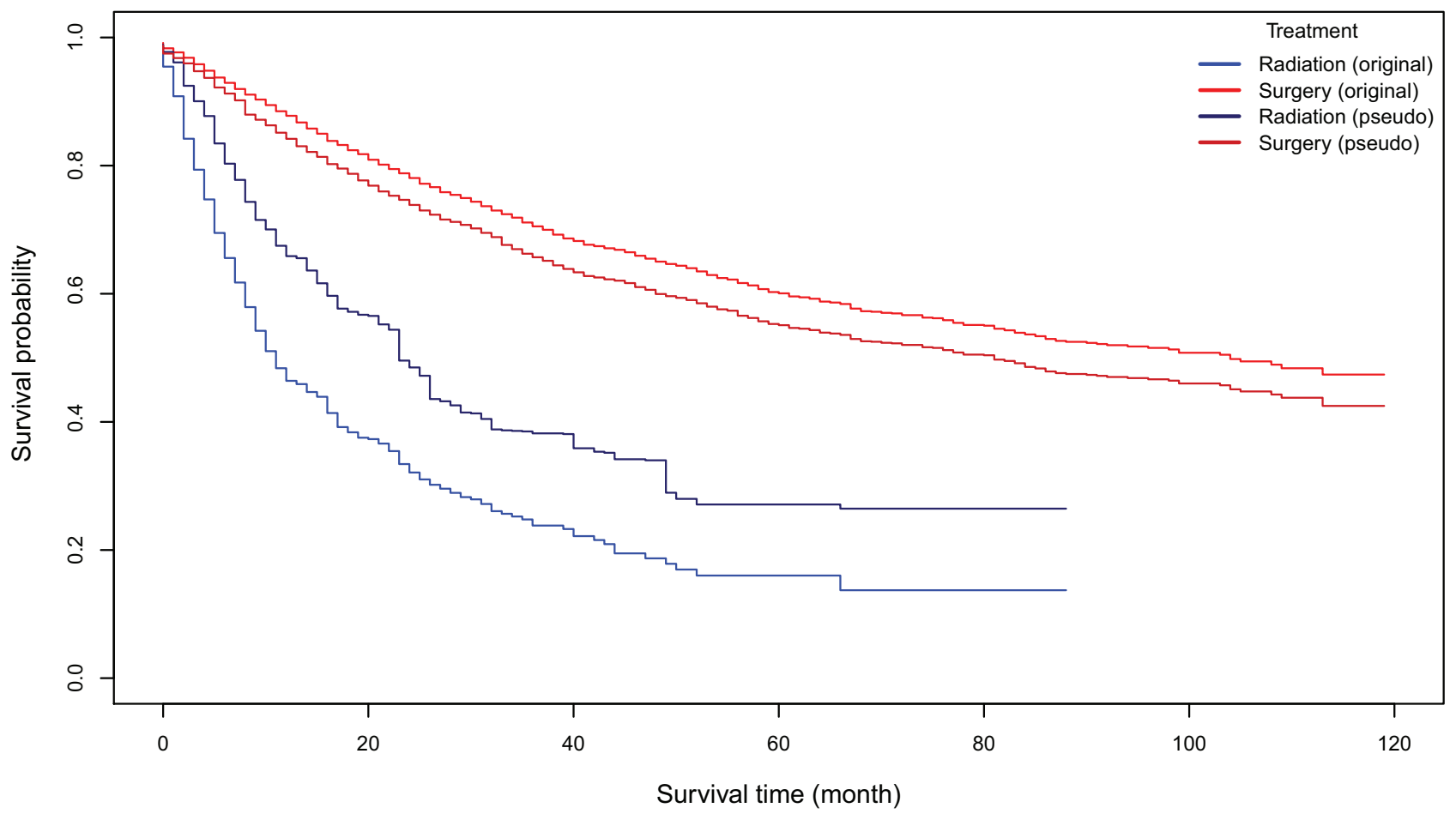

Figure I Overall and HCC-specific Kaplan-Meier survival curves for the original and pseudo samples. Panel (A) Overall Kaplan-Meier survival curves for the original and pseudo samples. Panel (B) HCC-specific Kaplan-Meier survival curves in the original and pseudo samples.

restricting the success of other treatment therapies, such as ablation, but less so for radiotherapy, suggesting that radiation may provide a unique therapeutic opportunity. ${ }^{23}$ While radiotherapy has emerged as an alternative treatment plan, however, debate still exists on which type of radiation therapy is superior. 
Table 3 Hazard Ratios, Confidence Internals, and P values Obtained from Cox Proportional Hazard Models for All-Cause Mortality and HCC-Specific Mortality in the Original Sample

\begin{tabular}{|c|c|c|c|c|}
\hline \multirow[t]{2}{*}{ Variable } & \multicolumn{2}{|c|}{ All-Cause Mortality } & \multicolumn{2}{|c|}{ HCC-Specific Mortality } \\
\hline & HR (95\% Cl) & P value & HR (95\% Cl) & $P$ value \\
\hline \multicolumn{5}{|l|}{ Treatment } \\
\hline Radiation & Reference & & Reference & \\
\hline Surgery & $0.48(0.43-0.54)$ & $<0.001$ & $0.44(0.38-0.50)$ & $<0.001$ \\
\hline \multicolumn{5}{|l|}{ Age } \\
\hline $20-39$ & Reference & & Reference & \\
\hline $40-59$ & $1.72(1.28-2.29)$ & $<0.001$ & $1.50(1.09-2.05)$ & 0.012 \\
\hline $60-79$ & $2.00(1.50-2.69)$ & $<0.001$ & $1.49(1.08-2.04)$ & 0.014 \\
\hline$\geq 80$ & $2.71(1.97-3.74)$ & $<0.001$ & $1.40(0.96-2.03)$ & 0.080 \\
\hline \multicolumn{5}{|l|}{ Gender } \\
\hline Female & Reference & & Reference & \\
\hline Male & $1.21(1.10-1.33)$ & $<0.001$ & $1.24(1.10-1.40)$ & $<0.001$ \\
\hline \multicolumn{5}{|l|}{ Race } \\
\hline White & Reference & & Reference & \\
\hline Black & $1.21(1.09-1.35)$ & $<0.001$ & $1.16(1.01-1.32)$ & 0.032 \\
\hline Chinese & $0.65(0.54-0.78)$ & $<0.001$ & $0.87(0.72-1.06)$ & 0.174 \\
\hline Others & $0.97(0.87-1.07)$ & 0.531 & $0.99(0.86-I . I 2)$ & 0.834 \\
\hline \multicolumn{5}{|l|}{ Marital status } \\
\hline Married/domestic partner & Reference & & Reference & \\
\hline Single (never married) & $1.27(1.15-1.42)$ & $<0.001$ & $1.32(1.16-1.50)$ & $<0.001$ \\
\hline Separated & $1.58(1.17-2.15)$ & 0.003 & $1.90(1.34-2.70)$ & $<0.001$ \\
\hline Divorced & $1.33(1.18-1.50)$ & $<0.001$ & $1.32(1.14-1.53)$ & $<0.001$ \\
\hline Widowed & $1.44(1.24-1.67)$ & $<0.001$ & $1.51(1.26-1.83)$ & $<0.001$ \\
\hline Other/unknown & $0.98(0.80-1.20)$ & 0.841 & $0.97(0.75-1.26)$ & 0.846 \\
\hline \multicolumn{5}{|l|}{ Tumor size $(\mathrm{cm})$} \\
\hline$<3.0$ & Reference & & Reference & \\
\hline $3.0-4.9$ & $1.42(1.28-1.57)$ & $<0.001$ & $1.47(1.29-1.67)$ & $<0.001$ \\
\hline $5.0-10.0$ & $1.49(1.32-1.68)$ & $<0.001$ & $1.57(1.35-1.83)$ & 0.000 \\
\hline$>10.0$ & $1.90(1.64-2.19)$ & $<0.001$ & $2.26(1.90-2.69)$ & $<0.001$ \\
\hline Unknown & $1.79(1.50-2.14)$ & $<0.001$ & $1.91(1.54-2.38)$ & $<0.001$ \\
\hline \multicolumn{5}{|l|}{ Grade } \\
\hline Grade I & Reference & & Reference & \\
\hline Grade II & $1.00(0.88-1.13)$ & 0.963 & I.I5 (0.98-I.36) & 0.084 \\
\hline Grade III & $1.41(1.21-1.65)$ & $<0.001$ & $1.78(1.47-2.16)$ & $<0.001$ \\
\hline Grade IV & $1.12(0.73-1.72)$ & 0.606 & $1.30(0.77-2.22)$ & 0.329 \\
\hline Unknown grade & $1.26(1.12-1.42)$ & $<0.001$ & $1.49(1.27-1.73)$ & $<0.001$ \\
\hline \multicolumn{5}{|l|}{ T stage } \\
\hline TI & Reference & & Reference & \\
\hline $\mathrm{T} 2$ & $1.12(1.01-1.24)$ & 0.027 & $1.14(1.01-1.29)$ & 0.039 \\
\hline $\mathrm{T} 3$ & $1.50(1.3 \mid-1.72)$ & $<0.001$ & $1.70(1.44-2.00)$ & $<0.001$ \\
\hline $\mathrm{T} 4$ & $1.37(1.07-1.75)$ & 0.012 & $1.32(0.99-1.77)$ & 0.056 \\
\hline $\mathrm{TX}$ & I.II (0.88-I.4I) & 0.387 & $1.19(0.90-1.57)$ & 0.222 \\
\hline \multicolumn{5}{|l|}{$N$ stage } \\
\hline No & Reference & & Reference & \\
\hline $\mathrm{NI}$ & $1.04(0.86-1.26)$ & 0.694 & I.I8 (0.95-I.45) & 0.127 \\
\hline
\end{tabular}

(Continued) 
Table 3 (Continued).

\begin{tabular}{|c|c|c|c|c|}
\hline \multirow[t]{2}{*}{ Variable } & \multicolumn{2}{|c|}{ All-Cause Mortality } & \multicolumn{2}{|c|}{ HCC-Specific Mortality } \\
\hline & HR (95\% Cl) & $P$ value & HR (95\% Cl) & $P$ value \\
\hline$N X$ & $1.30(1.11-1.53)$ & 0.001 & $1.39(1.15-1.67)$ & 0.001 \\
\hline \multicolumn{5}{|l|}{ M stage } \\
\hline Mo & Reference & & Reference & \\
\hline MI & $1.68(1.16-2.43)$ & 0.006 & $1.63(1.06-2.50)$ & 0.026 \\
\hline$M X$ & I. 15 (0.87-I.52) & 0.327 & $1.22(0.88-1.70)$ & 0.228 \\
\hline \multicolumn{5}{|c|}{ Disease extent condition } \\
\hline Localized & Reference & & Reference & \\
\hline Regional & $1.35(1.21-1.5 \mathrm{I})$ & $<0.001$ & $1.41(1.24-1.62)$ & $<0.001$ \\
\hline Distant & $1.72(1.18-2.49)$ & 0.005 & $1.91(1.24-2.96)$ & 0.003 \\
\hline Unstaged & $0.90(0.63-1.30)$ & 0.583 & $0.78(0.50-1.21)$ & 0.266 \\
\hline
\end{tabular}

Abbreviations: $\mathrm{HCC}$, hepatocellular carcinoma; HR, hazard ratio; $\mathrm{Cl}, 95 \%$ confidence interval.

Table 4 Hazard Ratios, Confidence Internals, and P values Obtained from Cox Proportional Hazard Models for All-Cause Mortality and HCC-Specific Mortality in the Pseudo-Sample

\begin{tabular}{|c|c|c|c|c|}
\hline \multirow[t]{2}{*}{ Variable } & \multicolumn{2}{|c|}{ All-Cause Mortality } & \multicolumn{2}{|c|}{ HCC-Specific Mortality } \\
\hline & HR $(95 \% \mathrm{Cl})$ & $P$ value & HR (95\% CI) & $P$ value \\
\hline \multicolumn{5}{|l|}{ Treatment } \\
\hline Radiation & Reference & & Reference & \\
\hline Surgery & $0.42(0.36-0.49)$ & $<0.001$ & $0.38(0.3 \mathrm{I}-0.46)$ & $<0.001$ \\
\hline \multicolumn{5}{|l|}{ Age } \\
\hline $20-39$ & Reference & & Reference & \\
\hline $40-59$ & $1.69(1.22-2.35)$ & 0.002 & $1.53(1.06-2.21)$ & 0.024 \\
\hline $60-79$ & $1.73(I .24-2.4 I)$ & 0.001 & $1.29(0.89-1.88)$ & 0.175 \\
\hline$\geq 80$ & 2.01 (1.38-2.92) & $<0.001$ & $0.96(0.60-1.5 I)$ & 0.846 \\
\hline \multicolumn{5}{|l|}{ Gender } \\
\hline Female & Reference & & Reference & \\
\hline Male & $1.27(1.08-1.50)$ & 0.005 & $1.23(0.99-1.5 \mathrm{I})$ & 0.062 \\
\hline \multicolumn{5}{|l|}{ Race } \\
\hline White & Reference & & & \\
\hline Black & $1.10(0.91-1.32)$ & 0.322 & $0.92(0.7|-| .2 \mid)$ & 0.564 \\
\hline Chinese & $0.7 \mid(0.44-1.15)$ & 0.164 & $0.96(0.57-1.64)$ & 0.890 \\
\hline Others & $0.83(0.67-1.01)$ & 0.063 & $0.79(0.62-1.02)$ & 0.066 \\
\hline \multicolumn{5}{|l|}{ Marital status } \\
\hline Married/domestic partner & Reference & & Reference & \\
\hline Single (never married) & $1.26(1.04-1.52)$ & 0.018 & $1.35(1.05-1.73)$ & 0.021 \\
\hline Separated & $1.36(0.81-2.29)$ & 0.248 & $1.74(1.03-2.94)$ & 0.039 \\
\hline Divorced & $1.26(1.05-1.52)$ & 0.015 & $1.32(1.05-1.65)$ & 0.018 \\
\hline Widowed & $1.42(1.17-1.74)$ & 0.001 & $1.48(1.14-1.92)$ & 0.003 \\
\hline Other/unknown & $1.16(0.81-1.66)$ & 0.408 & $0.73(0.46-1.17)$ & 0.190 \\
\hline \multicolumn{5}{|l|}{ Tumor size $(\mathrm{cm})$} \\
\hline$<3.0$ & Reference & & Reference & \\
\hline $3.0-4.9$ & $1.67(1.38-2.02)$ & $<0.001$ & $1.75(1.34-2.29)$ & $<0.001$ \\
\hline
\end{tabular}

(Continued) 
Table 4 (Continued).

\begin{tabular}{|c|c|c|c|c|}
\hline \multirow[t]{2}{*}{ Variable } & \multicolumn{2}{|c|}{ All-Cause Mortality } & \multicolumn{2}{|c|}{ HCC-Specific Mortality } \\
\hline & HR (95\% Cl) & $P$ value & HR (95\% CI) & $P$ value \\
\hline $5.0-10.0$ & I.8I (I.44-2.28) & $<0.001$ & $1.86(1.36-2.56)$ & $<0.001$ \\
\hline$>10.0$ & $2.17(1.62-2.91)$ & $<0.001$ & $2.54(1.77-3.66)$ & $<0.001$ \\
\hline Unknown & $2.33(1.72-3.16)$ & $<0.001$ & $2.55(I .7 \mid-3.80)$ & $<0.001$ \\
\hline \multicolumn{5}{|l|}{ Grade } \\
\hline Grade I & Reference & & Reference & \\
\hline Grade II & $0.90(0.69-1.18)$ & 0.437 & $1.14(0.78-1.68)$ & 0.492 \\
\hline Grade III & $1.49(1.09-2.05)$ & 0.012 & $1.81(1.18-2.76)$ & 0.006 \\
\hline Grade IV & $1.00(0.54-1.85)$ & 0.997 & $1.20(0.58-2.46)$ & 0.628 \\
\hline Unknown grade & $1.12(0.87-1.42)$ & 0.380 & $1.34(0.95-1.91)$ & 0.099 \\
\hline \multicolumn{5}{|l|}{ T stage } \\
\hline TI & Reference & & Reference & \\
\hline $\mathrm{T} 2$ & I.I7 (0.97-I.4I) & 0.101 & $1.14(0.87-1.49)$ & 0.348 \\
\hline T3 & $1.50(1.20-1.88)$ & $<0.001$ & $1.70(1.29-2.24)$ & $<0.001$ \\
\hline T4 & $2.03(1.10-3.73)$ & 0.023 & $2.13(1.05-4.32)$ & 0.035 \\
\hline TX & $0.92(0.64-1.33)$ & 0.655 & $0.93(0.60-1.42)$ & 0.722 \\
\hline \multicolumn{5}{|l|}{$\mathrm{N}$ stage } \\
\hline No & Reference & & Reference & \\
\hline $\mathrm{NI}$ & $0.75(0.55-1.02)$ & 0.068 & $0.82(0.59-1.15)$ & 0.250 \\
\hline NX & $1.28(0.97-1.69)$ & 0.087 & $1.44(1.04-2.01)$ & 0.029 \\
\hline \multicolumn{5}{|l|}{ M stage } \\
\hline Mo & Reference & & Reference & \\
\hline MI & $1.93(1.04-3.58)$ & 0.038 & $1.98(0.96-4.06)$ & 0.063 \\
\hline$M X$ & $0.93(0.58-1.50)$ & 0.760 & $0.93(0.54-1.60)$ & 0.781 \\
\hline \multicolumn{5}{|c|}{ Disease extent condition } \\
\hline Localized & Reference & & Reference & \\
\hline Regional & $1.23(1.03-1.48)$ & 0.024 & $1.22(0.97-1.53)$ & 0.097 \\
\hline Distant & $1.33(0.69-2.56)$ & 0.392 & $1.42(0.67-3.04)$ & 0.363 \\
\hline Unstaged & $0.89(0.52-1.52)$ & 0.664 & $0.72(0.38-1.36)$ & 0.305 \\
\hline
\end{tabular}

Abbreviations: HCC, hepatocellular carcinoma; HR: hazard ratio; $\mathrm{Cl}$ : $95 \%$ confidence interval.

In this study, a SEER registry sample of 5552 HCC patients was identified for the purpose of comparing overall survival and HCC-specific survival rates between patients who received surgery and patients who received radiotherapy. Of these 5552 patients, $82.8 \%$ received surgery, $9.7 \%$ received $\mathrm{BR}, 3.9 \%$ received RIT, and $3.5 \%$ received RI. Prior to IPTW, survival rates in the surgery group were superior to the radiation groups in both overall survival rate and HCC-specific survival rate. Cox proportional hazard regression both before and after IPTW confirmed that surgery was associated with higher survival rates compared with radiotherapy. Among the patients who received radiotherapy, those who received RIT or RI had better survival outcomes than those who received BR.
Despite the obvious benefits from surgery that have been reported previously, ${ }^{24,25}$ other studies using SEER registry data have found that nearly $50 \%$ of HCC patients who met Milan criteria ${ }^{26}$ for transplantation received only supportive care, and there was an apparent underutilization of surgical therapy in patients with HCC. ${ }^{27,28}$ In addition, Komatsu et $\mathrm{al}^{29}$ reported that particle radiotherapy is potentially preferable in HCC patients with stage IIIB inferior vena cava tumor thrombus and at least equal in efficiency to liver resection in those with stage IV disease.

For patients receiving radiation treatment, our study demonstrated a lower overall survival rate at the end of the $1^{\text {st }}$ year $(37.9 \%)$ than that reported in a previous study by Rim et al. ${ }^{30}$ McIntosh et $\mathrm{al}^{31}$ analyzed 20 patients with 


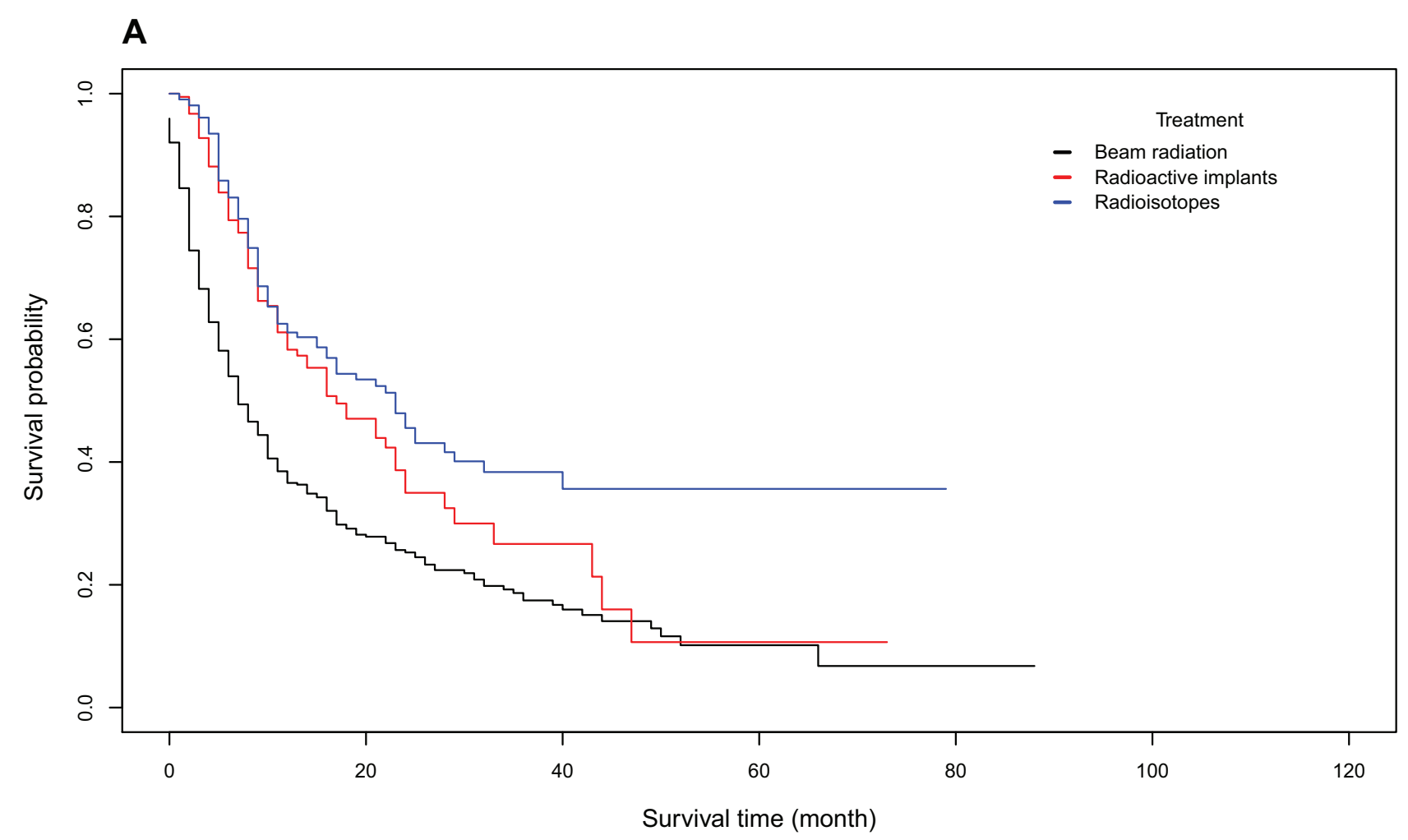

B

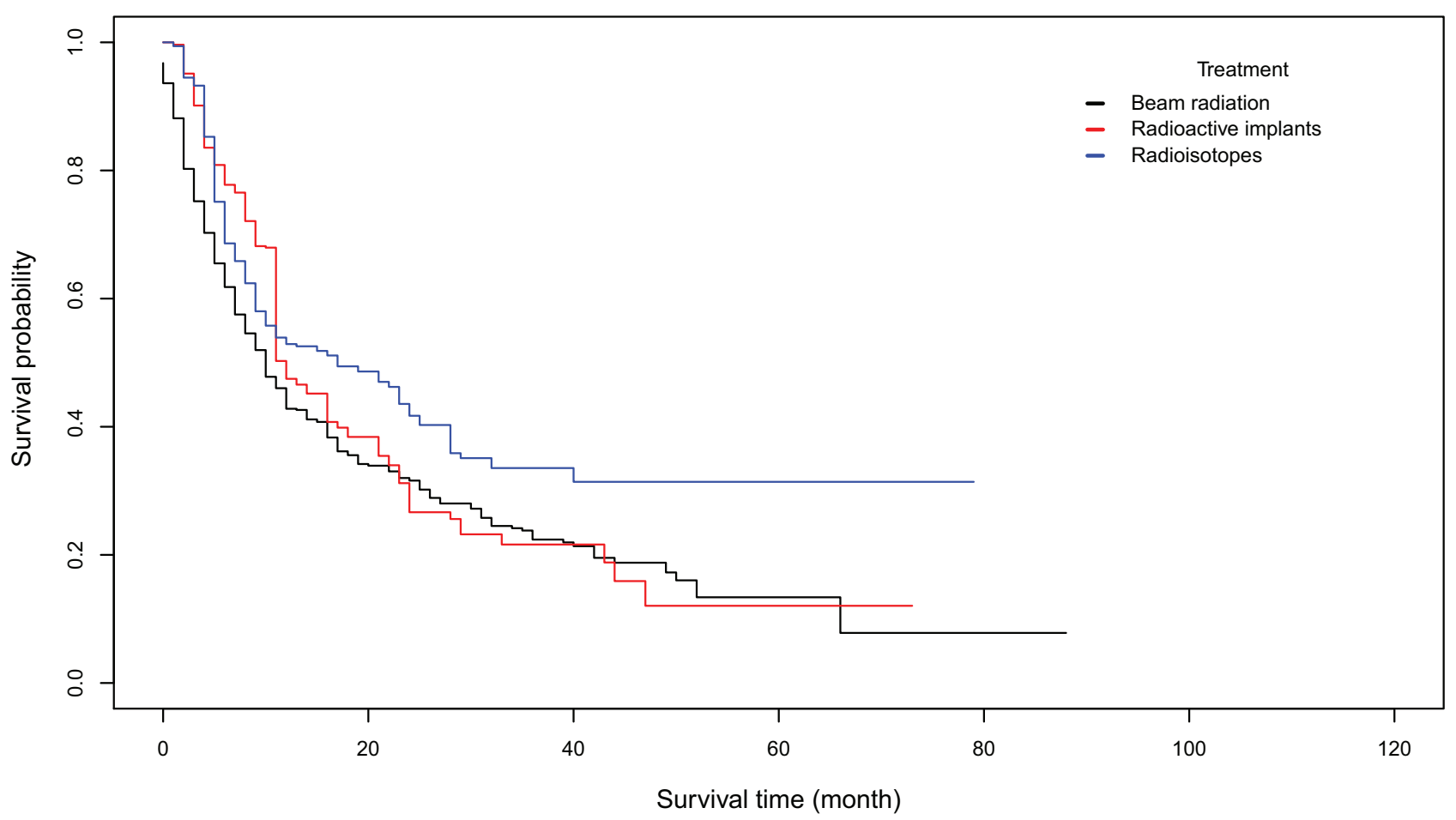

Figure 2 HCC-specific Kaplan-Meier survival curves for the 3 radiation groups in the original and pseudo samples. Panel (A) HCC-specific Kaplan-Meier survival curves for the 3 radiation groups in the original sample. Panel (B) HCC-specific Kaplan-Meier survival curves for the 3 radiation groups in the pseudo sample. 
Table 5 Hazard Ratios, Confidence Internals, and P values Obtained from Cox Proportional Hazard Models for All-Cause Mortality and HCC-Specific Mortality in the Original Radiation Sample

\begin{tabular}{|c|c|c|c|c|}
\hline \multirow[t]{2}{*}{ Variable } & \multicolumn{2}{|c|}{ All-Cause Mortality } & \multicolumn{2}{|c|}{ HCC-Specific Mortality } \\
\hline & HR (95\% Cl) & $P$ value & HR (95\% Cl) & $P$ value \\
\hline \multicolumn{5}{|l|}{ Treatment } \\
\hline BR & Reference & & Reference & \\
\hline RI & $0.86(0.68-1.08)$ & 0.191 & $0.85(0.65-1.11)$ & 0.227 \\
\hline RIT & $0.68(0.54-0.87)$ & 0.002 & $0.69(0.52-0.91)$ & 0.008 \\
\hline \multicolumn{5}{|l|}{ Age } \\
\hline $20-39$ & Reference & & Reference & \\
\hline $40-59$ & $1.34(0.72-2.50)$ & 0.358 & $1.23(0.64-2.38)$ & 0.539 \\
\hline $60-79$ & $1.10(0.59-2.06)$ & 0.767 & $0.88(0.45-1.7 I)$ & 0.707 \\
\hline$\geq 80$ & $1.09(0.55-2.16)$ & 0.816 & $0.68(0.32-1.46)$ & 0.322 \\
\hline \multicolumn{5}{|l|}{ Gender } \\
\hline Female & Reference & & Reference & \\
\hline Male & I.II (0.89-I.38) & 0.374 & $1.15(0.89-1.50)$ & 0.280 \\
\hline \multicolumn{5}{|l|}{ Race } \\
\hline White & Reference & & Reference & \\
\hline Black & $1.20(0.97-1.49)$ & 0.095 & $1.04(0.81-1.33)$ & 0.762 \\
\hline Chinese & $0.86(0.49-1.53)$ & 0.617 & $1.09(0.60-1.99)$ & 0.773 \\
\hline Others & $1.03(0.81-1.32)$ & 0.803 & $0.93(0.70-1.24)$ & 0.634 \\
\hline \multicolumn{5}{|l|}{ Marital status } \\
\hline Married/domestic partner & Reference & & Reference & \\
\hline Single (never married) & $0.92(0.74-1.14)$ & 0.448 & $0.90(0.7 I-1.15)$ & 0.413 \\
\hline Separated & $1.61(0.65-4.03)$ & 0.306 & $2.25(0.89-5.68)$ & 0.086 \\
\hline Divorced & $1.12(0.85-1.48)$ & 0.424 & $1.19(0.88-1.62)$ & 0.261 \\
\hline Widowed & $1.16(0.81-1.67)$ & 0.426 & $1.17(0.76-1.80)$ & 0.485 \\
\hline Other/unknown & $0.97(0.60-1.56)$ & 0.886 & $0.69(0.36-1.31)$ & 0.254 \\
\hline \multicolumn{5}{|l|}{ Tumor size $(\mathrm{cm})$} \\
\hline$<3.0$ & Reference & & Reference & \\
\hline $3.0-4.9$ & $1.70(1.27-2.28)$ & $<0.001$ & $1.68(1.19-2.37)$ & 0.003 \\
\hline $5.0-10.0$ & $1.78(1.32-2.4 I)$ & $<0.001$ & $1.69(1.19-2.39)$ & 0.003 \\
\hline$>10.0$ & $2.10(1.49-2.95)$ & $<0.001$ & $1.95(1.31-2.90)$ & 0.001 \\
\hline Unknown & $2.47(1.74-3.50)$ & $<0.001$ & $2.30(1.54-3.43)$ & $<0.001$ \\
\hline \multicolumn{5}{|l|}{ Grade } \\
\hline Grade I & Reference & & Reference & \\
\hline Grade II & $0.78(0.56-1.08)$ & 0.136 & $0.98(0.66-1.45)$ & 0.907 \\
\hline Grade III & $1.31(0.90-1.90)$ & 0.154 & $1.58(1.03-2.45)$ & 0.038 \\
\hline Grade IV & $1.33(0.17-10.23)$ & 0.783 & $1.99(0.25-15.67)$ & 0.515 \\
\hline Unknown grade & $0.95(0.72-1.25)$ & 0.698 & $1.08(0.77-1.53)$ & 0.647 \\
\hline \multicolumn{5}{|l|}{ T stage } \\
\hline TI & Reference & & Reference & \\
\hline $\mathrm{T} 2$ & $1.23(0.96-1.59)$ & 0.106 & $1.16(0.86-1.57$ & 0.338 \\
\hline $\mathrm{T} 3$ & $1.43(1.12-1.82)$ & 0.004 & $1.56(1.18-2.06)$ & 0.002 \\
\hline $\mathrm{T} 4$ & $0.76(0.50-1.15)$ & 0.198 & $0.79(0.49-1.27)$ & 0.332 \\
\hline $\mathrm{TX}$ & $0.86(0.62-1.20)$ & 0.371 & $0.90(0.62-1.30)$ & 0.565 \\
\hline \multicolumn{5}{|l|}{$N$ stage } \\
\hline No & Reference & & Reference & \\
\hline
\end{tabular}

(Continued) 
Table 5 (Continued).

\begin{tabular}{|c|c|c|c|c|}
\hline \multirow[t]{2}{*}{ Variable } & \multicolumn{2}{|c|}{ All-Cause Mortality } & \multicolumn{2}{|c|}{ HCC-Specific Mortality } \\
\hline & HR (95\% Cl) & $P$ value & HR (95\% CI) & $P$ value \\
\hline NI & $1.06(0.82-1.38)$ & 0.644 & $1.12(0.84-1.49)$ & 0.444 \\
\hline$N X$ & $1.43(1.13-1.82)$ & 0.003 & $1.47(1.13-1.91)$ & 0.004 \\
\hline \multicolumn{5}{|l|}{ M stage } \\
\hline Mo & Reference & & Reference & \\
\hline MI & $0.94(0.42-2.10)$ & 0.880 & $0.82(0.35-1.95)$ & 0.661 \\
\hline$M X$ & $0.98(0.58-1.66)$ & 0.954 & $0.99(0.54-1.80)$ & 0.965 \\
\hline \multicolumn{5}{|c|}{ Disease extent condition } \\
\hline Localized & Reference & & Reference & \\
\hline Regional & $1.17(0.91-1.50)$ & 0.212 & I.I2(0.83-I.49) & 0.464 \\
\hline Distant & $2.69(1.18-6.12)$ & 0.018 & $3.28(1.35-7.95)$ & 0.009 \\
\hline Unstaged & $0.80(0.37-1.74)$ & 0.568 & $0.81(0.33-1.98)$ & $0.64 I$ \\
\hline
\end{tabular}

Abbreviations: HCC, hepatocellular carcinoma; HR, hazard ratio; $\mathrm{Cl}, 95 \%$ confidence interval; BR, beam radiation; RI, radioactive implants; RIT, radioisotopes.

unresectable HCC who underwent helical tomotherapybased intensity-modulated radiation therapy (IMRT) and reported 1-year overall survival rates of $73 \%$ and $11 \%$ for patients with Child-Pugh classification A and B disease, respectively. In another study conducted by Lo et al, ${ }^{32} 53$ patients with unresectable $\mathrm{HCC}$ and a median tumor size of $4.3 \mathrm{~cm}$ who received stereotactic body radiotherapy presented with a median overall survival rate of 20 months. When compared with our results, the higher survival rates in these studies may be explained by the fact that the SEER registry data utilized in our study represented a mixture of various radiation treatments.

Analysis results from the pseudo-sample generated from our IPTW approach validated our findings in the original sample. However, the weighted 5-year survival rate of $40 \%$ in the surgery group was below the range of the 5-year survival rates of $62.1 \%$ to $91.5 \%$ reported in previous studies. ${ }^{1,33-38}$ In the radiation group, the overall weighted survival rate at the end of the $5^{\text {th }}$ year was only $15.8 \%$, which was below the range of the 5-year survival rates of $66 \%$ to $85.9 \%$ reported in previous studies. ${ }^{1}$ As many studies have shown, the variability of overall survival rates among patients who receive radiation may be accounted for by the subtypes and dose employed during radiotherapy. Jang et $\mathrm{al}^{39}$ demonstrated that a 2-year overall survival rate for patients who received radiation dosages of $<45,45-54$, and $>54$ Gray were $71 \%, 64 \%$, and $30 \%$, respectively.

In our original radiation subgroups, prior to IPTW, the RIT group was superior to the BR group in terms of overall survival rate and HCC-specific survival rate, but there was no significant difference between the RI and BR groups. In the radiation pseudo-sample, both the RIT and RI groups outperformed the BR group in overall survival. Similar results were discovered in HCC-specific survival rates between these groups. Our study demonstrated a median overall survival time of 6 months in the BR group, which was shorter than the overall survival times reported in some other studies. ${ }^{40-43}$ The median overall survival time of the RIT group in our study was 14 months, which was longer than that reported by Mercedes et $\mathrm{al}^{44}$ but shorter than that reported by Kulik et al. ${ }^{45}$

Our investigation confirms that propensity score IPTW is an efficient and helpful method of creating balanced groups to assess the effects of different therapies, although there are still some limitations in this study. Our study shows that surgery is significantly associated with improved overall and HCC-specific survival rates among SEER patients with HCC. However, selection of HCC treatments may largely depend on patients' clinical characteristics, and therefore clinicians should not choose one treatment over another for an HCC patient based on the conclusions drawn from this study. The decision of treatment of $\mathrm{HCC}$ is a selection-procedure by clinicians according to the Clinical Practice Guidelines for HCC. ${ }^{46,47}$ Another limitation of our study is that our data from the SEER registry did not include any critical details on surgery and radiation therapy, consequently restricting our clinical investigation. For instance, when a patient received surgery as the HCC treatment, the SEER registry did not record whether the surgical procedure was partial 
Table 6 Hazard Ratios, Confidence Internals, and P values Obtained from Cox Proportional Hazard Models for All-Cause Mortality and HCC-Specific Mortality in the Radiation Pseudo-Sample

\begin{tabular}{|c|c|c|c|c|}
\hline \multirow[t]{2}{*}{ Variable } & \multicolumn{2}{|c|}{ All-Cause Mortality } & \multicolumn{2}{|c|}{ HCC-Specific Mortality } \\
\hline & HR (95\% CI) & $P$ value & HR (95\% CI) & $P$ value \\
\hline \multicolumn{5}{|l|}{ Treatment } \\
\hline BR & Reference & & Reference & \\
\hline RI & $0.80(0.7 \mathrm{I}-0.90)$ & $<0.001$ & $0.84(0.73-0.96)$ & 0.010 \\
\hline RIT & $0.79(0.70-0.90)$ & $<0.001$ & $0.83(0.72-0.96)$ & 0.010 \\
\hline \multicolumn{5}{|l|}{ Age } \\
\hline $20-39$ & Reference & & Reference & \\
\hline $40-59$ & $1.99(1.22-3.24)$ & 0.005 & $1.49(0.90-2.45)$ & 0.119 \\
\hline $60-79$ & $1.33(0.8 \mathrm{I}-2.17)$ & 0.257 & $0.89(0.53-1.47)$ & 0.639 \\
\hline$\geq 80$ & $1.40(0.82-2.38)$ & 0.216 & $0.82(0.47-1.45)$ & 0.501 \\
\hline \multicolumn{5}{|l|}{ Gender } \\
\hline Female & Reference & & Reference & \\
\hline Male & $1.20(1.03-1.39)$ & 0.016 & $1.37(1.15-1.64)$ & 0.001 \\
\hline \multicolumn{5}{|l|}{ Race } \\
\hline White & Reference & & Reference & \\
\hline Black & $1.14(0.98-1.33)$ & 0.079 & $1.04(0.87-1.24)$ & 0.646 \\
\hline Chinese & $0.87(0.53-1.44)$ & 0.597 & $1.08(0.64-1.83)$ & 0.765 \\
\hline Others & $1.15(0.97-1.36)$ & 0.107 & $1.09(0.89-1.32)$ & 0.406 \\
\hline \multicolumn{5}{|l|}{ Marital status } \\
\hline Married/domestic partner & Reference & & Reference & \\
\hline Single (never married) & $0.90(0.78-1.04)$ & 0.167 & $0.91(0.77-1.08)$ & 0.281 \\
\hline Separated & $1.34(0.66-2.73)$ & 0.414 & $1.78(0.87-3.65)$ & 0.113 \\
\hline Divorced & $1.10(0.91-1.33)$ & 0.341 & $1.21(0.98-|.5|)$ & 0.077 \\
\hline Widowed & $1.16(0.91-1.47)$ & 0.220 & $1.06(0.79-1.42)$ & 0.697 \\
\hline Other/unknown & $1.22(0.82-1.80)$ & 0.327 & $0.69(0.38-1.25)$ & 0.223 \\
\hline \multicolumn{5}{|l|}{ Tumor size $(\mathrm{cm})$} \\
\hline$<3.0$ & Reference & & Reference & \\
\hline $3.0-4.9$ & $1.88(1.54-2.28)$ & $<0.001$ & $1.83(1.46-2.29)$ & $<0.001$ \\
\hline $5.0-10.0$ & $1.65(1.35-2.01)$ & $<0.001$ & $1.40(1.11-1.77)$ & 0.004 \\
\hline$>10.0$ & $2.31(1.84-2.90)$ & $<0.001$ & $1.97(1.52-2.56)$ & $<0.001$ \\
\hline Unknown & $2.58(2.02-3.29)$ & $<0.001$ & $2.26(1.71-2.99)$ & $<0.001$ \\
\hline \multicolumn{5}{|l|}{ Grade } \\
\hline Grade I & Reference & & Reference & \\
\hline Grade II & $0.73(0.59-0.90)$ & 0.004 & $1.00(0.78-1.28)$ & 0.971 \\
\hline Grade III & $1.07(0.84-1.36)$ & 0.605 & $1.28(0.96-1.70)$ & 0.089 \\
\hline Grade IV & $1.81(0.45-7.30)$ & 0.407 & $4.12(1.00-17.01)$ & 0.050 \\
\hline Unknown grade & $0.90(0.76-1.07)$ & 0.247 & $1.02(0.82-1.27)$ & 0.840 \\
\hline \multicolumn{5}{|l|}{ T stage } \\
\hline TI & Reference & & Reference & \\
\hline $\mathrm{T} 2$ & $1.27(1.07-1.50)$ & 0.006 & $1.34(1.10-1.65)$ & 0.004 \\
\hline T3 & $1.70(1.44-2.00)$ & $<0.001$ & $2.19(1.81-2.64)$ & $<0.001$ \\
\hline $\mathrm{T} 4$ & $0.94(0.69-1.28)$ & 0.699 & $\mathrm{I} .13(0.79-1.6 \mathrm{I})$ & 0.518 \\
\hline $\mathrm{TX}$ & $1.01(0.79-1.30)$ & 0.916 & I.0I $(0.76-1.36)$ & 0.929 \\
\hline \multicolumn{5}{|l|}{$\mathrm{N}$ stage } \\
\hline No & Reference & & Reference & \\
\hline
\end{tabular}

(Continued) 
Table 6 (Continued).

\begin{tabular}{|c|c|c|c|c|}
\hline \multirow[t]{2}{*}{ Variable } & \multicolumn{2}{|c|}{ All-Cause Mortality } & \multicolumn{2}{|c|}{ HCC-Specific Mortality } \\
\hline & HR (95\% CI) & $P$ value & HR (95\% CI) & $P$ value \\
\hline $\mathrm{NI}$ & $0.79(0.66-0.95)$ & 0.012 & $0.62(0.50-0.77)$ & $<0.001$ \\
\hline NX & $1.31(1.10-1.55)$ & 0.002 & $1.45(1.20-1.74)$ & $<0.001$ \\
\hline \multicolumn{5}{|l|}{ M stage } \\
\hline Mo & Reference & & Reference & \\
\hline MI & $1.70(1.04-2.79)$ & 0.035 & $1.57(0.9 \mid-2.7 I)$ & 0.108 \\
\hline$M X$ & $1.15(0.83-1.59)$ & 0.396 & $1.04(0.72-1.52)$ & 0.818 \\
\hline \multicolumn{5}{|c|}{ Disease extent condition } \\
\hline Localized & Reference & & Reference & \\
\hline Regional & $1.17(1.00-1.36)$ & 0.049 & $1.07(0.89-1.28)$ & 0.468 \\
\hline Distant & $1.65(0.99-2.73)$ & 0.053 & $2.12(1.21-3.72)$ & 0.008 \\
\hline Unstaged & $0.76(0.45-1.28)$ & 0.301 & $0.89(0.49-1.63)$ & 0.705 \\
\hline
\end{tabular}

Abbreviations: HCC, hepatocellular carcinoma; HR, hazard ratio; $\mathrm{Cl}, 95 \%$ confidence interval; BR, beam radiation; RI, radioactive implants; RIT, radioisotopes.

hepatectomy, transplant, wedge resection, or lobectomy. Likewise, when a patient received radiotherapy, the SEER registry did not record radiation dose or other details regarding the radiotherapy. Lastly, the data analysis method of propensity score balancing was invented to mimic randomization by creating a pseudo-sample in which the subjects in two or more treatment groups are comparable. However, this method relies on the assumption of "strong ignorability," which requires that there be no unmeasured confounders. In our study, due to the limitations inherited from the SEER registry, there must be some covariates that were not measured on HCC patients. Therefore, our analysis of the surgery and radiotherapy groups was not to derive causality but to confirm a population-level association.

\section{Conclusion}

This study demonstrated that SEER registry HCC patients who received surgery as their HCC treatment had a better survival rate compared with those who received radiotherapy. We evaluated both overall survival and $\mathrm{HCC}$-specific survival rates, before and after propensity score weighting. This study also discovered that among patients who received radiotherapy, those who received RIT and RI outperformed those who received BR in terms of overall survival and HCC-specific survival rates after IPTW.

\section{Author Contributions}

All authors made substantial contributions to conception and design, acquisition of data, or analysis and interpretation of data; took part in drafting the article or revising it critically for important intellectual content; agreed to submit to the current journal; gave final approval of the version to be published; and agree to be accountable for all aspects of this research work. Dr. Fuyan Shi, Chen Wang, Dr. Yujia Kong made equal contribution to this research article. Dr. Bo Zhang and Dr. Suzhen Wang are co-senior authors of this research article.

\section{Funding}

Dr. Fuyan Shi's research was partially supported by the National Natural Science Foundation of China (No. 81803337), the Shandong Provincial Youth Innovation Team Development Plan of Colleges and Universities (No.2019-6-156, Lu-Jiao), the Shandong Provincial Government Fund for Overseas Study (No. 27, 2019, LuJiao), the Shandong Science and Technology Development Plan Project (No. 2015 WS0067), and the Weifang Medical University Doctoral Foundation Project (No. 2017BSQD51). Liping Yang's research was partially supported by the Shaanxi Key Industry Innovation Chain (No. 2016KTZDSF02-07-01). Dr. Suzhen Wang's research was partially supported by the National Natural Science Foundation of China (No. 81872719), the National Bureau of Statistics Foundation Project (No. 2018LY79), the Natural Science Foundation of Shandong Province (No. 2019MH034), and the Poverty Alleviation Fund project of Weifang Medical University (No. FP1801001).

\section{Disclosure}

The authors declare that they have no conflict of interests. 


\section{References}

1. Takayasu K, Arii S, Sakamoto M, et al. Impact of resection and ablation for single hypovascular hepatocellular carcinoma $<=2 \mathrm{~cm}$ analysed with propensity score weighting. Liver Int. 2018;38:484493.

2. El-Serag HB. Epidemiology of viral hepatitis and hepatocellular carcinoma. Gastroenterology. 2012;142(6):e1261. doi:10.1053/j. gastro.2011.12.061

3. Gray SH, White JA, Li P. A SEER Database Analysis of the Survival Advantage of Transarterial Chemoembolization for Hepatocellular Carcinoma: an Underutilized Therapy. $J$ Vasc Interv Radiol. 2017;28(2):e232. doi:10.1016/j.jvir.2016.09.022

4. Su M, Zhao Y, Liu J. The role of definitive local treatment in metastatic hepatocellular carcinoma patients: A SEER-based study. Medicine. 2018;97(10):e0020. doi:10.1097/MD.0000000000010020

5. Hirano K, Imbens GW. Estimation of causal effects using propensity score weighting_An application to data on right heart catheterization. Health Serv Outcomes Res Methodol. 2001;2:259-278.

6. Hirano K, Imbens GW, Ridder G. Efficient estimation of average treatment effects using the estimated propensity score. Econometrica. 2003;71:1161-1189.

7. Vakulenko-Lagun B, Mandel M, Betensky RA. Inverse probability weighting methods for Cox regression with right-truncated data Biometrics. 2019;2:154.

8. Harlan LCHBF. The surveillance, epidemiology, and end-results program database as a resource for conducting descriptive epidemiologic and clinical studies. J Clin Oncol. 2003;2232-2233.

9. A PC F, Jack A, Shanmugaratnam K, Sobin L, Parkin DM, Whelan S. International Classification of Diseases for Oncology. 3 ed. Geneva: World Health Organization; 2000.

10. McCaffrey DF, Griffin BA, Almirall D, Slaughter ME, Ramchand R, Burgette LF. A tutorial on propensity score estimation for multiple treatments using generalized boosted models. Stat Med. 2013;32 (19):3388-3414. doi:10.1002/sim.5753

11. Parast L, McCaffrey DF, Burgette LF, et al. Optimizing VarianceBias Trade-off in the TWANG Package for Estimation of Propensity Scores. Health Serv Outcomes Res Methodol. 2017;17(3-4):175-197. doi:10.1007/s10742-016-0168-2

12. Burgette JM, Preisser JS, Rozier RG. Rozier RG.Propensity score weighting: an application to an Early Head Start dental study. Journal of Public Health Dentistry. 2016;76(1):17-29. doi:10.1111/ jphd.12106

13. Feng P, Zhou X-H, Zou Q-M, Fan M-Y, Li X-S. Generalized propensity score for estimating the average treatment effect of multiple treatments. Stat Med. 2012;31(7):681-697. doi:10.1002/sim.4168

14. Ridgeway G, McCaffrey D, Griffin BA, Burgette L. "Twang: toolkit for weighting and analysis of non-equivalent groups." Available at http:// cran.rproject.org/web/packages/twang/vignettes/twang.pdf. 2014.

15. JM HM R, Brumback B. Marginal structural models and causal inference in epidemiology. Epidemiology. 2000;550-560.

16. Cole SR, Hernán MA. Hernan MA: adjusted survival curves with inverse probability weights. Comput Methods Programs Biomed. 2004;75(1):45-49. doi:10.1016/j.cmpb.2003.10.004

17. Cole SR, Hernan MA. Constructing inverse probability weights for marginal structural models. Am J Epidemiol. 2008;168(6):656-664. doi:10.1093/aje/kwn164

18. Xie J, Liu C. Adjusted Kaplan-Meier estimator and log-rank test with inverse probability of treatment weighting for survival data. Stat Med. 2005;24(20):3089-3110. doi:10.1002/sim.2174

19. Dunkler D, Ploner M, Schemper M, Heinze G. Weighted Cox Regression Using the R Package coxphw. J Stat Softw. 2018;84(2). doi:10.18637/jss.v084.i02

20. Le Borgne F. Package 'IPWsurvival': propensity Score Based Adjusted Survival Curves and Corresponding Log-Rank Statistic https://CRANR-projectorg/package=IPWsurvival. 2017.
21. Khan K. Colorectal cancer with liver metastases: neoadjuvant chemotherapy, surgical resection first or palliation alone? World $J$ Gastroenterol. 2014;20(35):12391-12406. doi:10.3748/wjg.v20. i35.12391

22. Grundmann RT. Current state of surgical treatment of liver metastases from colorectal cancer. World J Gastrointest Surg. 2011;3 (12):183-196. doi:10.4240/wjgs.v3.i12.183

23. Wigg AJ, Palumbo K, Wigg DR. Wigg DR: radiotherapy for hepatocellular carcinoma: systematic review of radiobiology and modeling projections indicate reconsideration of its use. $J$ Gastroenterol Hepatol. 2010;25(4):664-671. doi:10.1111/j.1440-1746.2009.06126. $\mathrm{x}$

24. Mokdad AA, Hester CA, Singal AG, Yopp AC. Management of hepatocellular in the United States. Chinese Clinical Oncology. 2017;6(2):21. doi:10.21037/cco.2017.04.04

25. Khan AS. Current surgical treatment strategies for hepatocellular carcinoma in North America. World J Gastroenterol. 2014;20 (41):15007-15017. doi:10.3748/wjg.v20.i41.15007

26. Wald C, Russo MW, Heimbach JK, Hussain HK, Pomfret EA, Bruix J. New OPTN/UNOS Policy for Liver Transplant Allocation: standardization of Liver Imaging, Diagnosis, Classification, and Reporting of Hepatocellular Carcinoma. Radiology. 2013;266 (2):376-382. doi:10.1148/radiol.12121698

27. Devaki P, Wong RJ, Marupakula V, et al. Approximately one-half of patients with early-stage hepatocellular carcinoma meeting Milan criteria did not receive local tumor destructive or curative surgery in the post-MELD exception era. Cancer. 2014;120(11):1725-1732. doi:10.1002/cncr.28639

28. Nathan H, Hyder O, Mayo SC, et al. Surgical therapy for early hepatocellular carcinoma in the modern era: a 10-year SEER-medicare analysis. Ann Surg. 2013;258(6):1022-1027. doi:10.1097/ SLA.0b013e31827da749

29. Komatsu S, Kido M, Asari S, et al. Particle radiotherapy, a novel external radiation therapy, versus liver resection for hepatocellular carcinoma accompanied with inferior vena cava tumor thrombus: A matched-pair analysis. Surgery. 2017;162(6):1241-1249. doi:10.1016/j.surg.2017.08.006

30. Rim CH, Kim CY, Yang DS, Yoon WS. Comparison of radiation therapy modalities for hepatocellular carcinoma with portal vein thrombosis: A meta-analysis and systematic review. Radiother Oncol. 2018;129(1):112-122. doi:10.1016/j.radonc.2017.11.013

31. McIntosh A, Hagspiel KD, Al-Osaimi AM, et al. Accelerated treatment using intensity-modulated radiation therapy plus concurrent capecitabine for unresectable hepatocellular carcinoma. Cancer. 2009;115(21):5117-5125. doi:10.1002/cncr.24552

32. Lo C-H, Huang W-Y, Lee M-S, et al. Stereotactic ablative radiotherapy for unresectable hepatocellular carcinoma patients who failed or were unsuitable for transarterial chemoembolization. Eur $J$ Gastroenterol Hepatol. 2014;26(3):345-352. doi:10.1097/ MEG.0000000000000032

33. Wang J-H, Wang -C-C, Hung C-H, Chen C-L, Lu S-N. Survival comparison between surgical resection and radiofrequency ablation for patients in BCLC very early/early stage hepatocellular carcinoma. J Hepatol. 2012;56(2):412-418. doi:10.1016/j.jhep.2011.05.020

34. Zhen-Wei Peng Z-W, Lin X-J, Zhang Y-J. Radiofrequency Ablation versus Hepatic Resection for the Treatment of Hepatocellular Carcinomas $2 \mathrm{~cm}$ or Smaller: A Retrospective Comparative Study. Radiology. 2012;262(3):1022-1033. doi:10.1148/radiol.11110817

35. Hasegawa K, Kokudo N, Makuuchi M, et al. Comparison of resection and ablation for hepatocellular carcinoma: a cohort study based on a Japanese nationwide survey. J Hepatol. 2013;58(4):724-729. doi:10.1016/j.jhep.2012.11.009

36. Liu PH, Hsu CY, Hsia CY, et al. Surgical Resection Versus Radiofrequency Ablation for Single Hepatocellular Carcinoma $<=$ $2 \mathrm{~cm}$ in a Propensity Score Model. Ann Surg. 2016;263:538-545. 
37. Kim G-A, Shim JH, Kim M-J, et al. Radiofrequency ablation as an alternative to hepatic resection for single small hepatocellular carcinomas. Br J Surg. 2016;103(1):126-135. doi:10.1002/bjs.9960

38. Roayaie S, Obeidat K, Sposito C, et al. Resection of hepatocellular cancer $\leq 2 \mathrm{~cm}$ : results from two Western centers. Hepatology. 2013;57 (4):1426-1435. doi:10.1002/hep.25832

39. Won Il Jang WI, Kim M-S, Bae SH. High-dose stereotactic body radiotherapy correlates increased local control and overall survival in patients with inoperable hepatocellular carcinoma. Radiation Oncology. 2013;8(1):250. doi:10.1186/1748-717X-8-250

40. Berger NG, Tanious MN, Hammad AY, et al. External radiation or ablation for solitary hepatocellular carcinoma: A survival analysis of the SEER database. J Surg Oncol. 2016;76(3):307-312. doi:10.1002/ jso. 24661

41. Bujold A, Massey CA, Kim JJ, et al. Sequential Phase I and II trials of stereotactic body radiotherapy for locally advanced hepatocellular carcinoma. J Clin Oncol. 2013;31:1631-1639.

42. Lasley FD, Mannina EM, Johnson CS, et al. Treatment variables related to liver toxicity in patients with hepatocellular carcinoma, Child-Pugh class A and B enrolled in a Phase 1-2 trial of stereotactic body radiation therapy. Pract Radiat Oncol. 2015;5:e443-e449.
43. Scorsetti M, Comito T, Cozzi L, et al. The challenge of inoperable hepatocellular carcinoma (HCC): results of a single-institutional experience on stereotactic body radiation therapy (SBRT). J Cancer Res Clin Oncol. 2015;141:1301-1309.

44. Inarrairaegui $\mathrm{M}$, Thurston $\mathrm{KG}$, Bilbao JI, et al. Radioembolization with use of yttrium-90 resin microspheres in patients with hepatocellular carcinoma and portal vein thrombosis. J Vasc Interv Radiol. 2010;21:1205-1212.

45. Kulik LM, Carr BI, Mulcahy MF, et al. Safety and efficacy of $90 \mathrm{Y}$ radiotherapy for hepatocellular carcinoma with and without portal vein thrombosis. Hepatology. 2008;47:71-81.

46. European Association for the Study of the Liver. EASL Clinical Practice Guidelines: management of hepatocellular carcinoma. $J$ Hepatol. 2018;69:182-236.

47. Vogel A, Cervantes A, Chau I, et al. Hepatocellular carcinoma: ESMO Clinical Practice Guidelines for diagnosis, treatment and follow-up. Annals of Oncology. 2019;30(5):871-873.

\section{Publish your work in this journal}

The Journal of Hepatocellular Carcinoma is an international, peerreviewed, open access journal that offers a platform for the dissemination and study of clinical, translational and basic research findings in this rapidly developing field. Development in areas including, but not limited to, epidemiology, vaccination, hepatitis therapy, pathology and molecular tumor classification and prognostication are all considered for publication. The manuscript management system is completely online and includes a very quick and fair peer-review system, which is all easy to use. Visit http://www.dovepress.com/ testimonials.php to read real quotes from published authors. 